\begin{tabular}{|c|c|}
\hline Title & A naly sis of Litz Wire L osses U sing Homogenization-Based FEM \\
\hline Author(s) & Otomo, Y oshitsugu; Igarashi, Hajime; Sano, Hiroyuki; Y amada, T akashi \\
\hline Citation & $\begin{array}{l}\text { IEEE Transactions on Magnetics, 57(8), } 7402409 \\
\text { https://doi.org/10.1109/ MA G.2021.3081819 }\end{array}$ \\
\hline Issue Date & $2021-08$ \\
\hline Doc URL & http:/hdl.handle.net/2115/82297 \\
\hline Rights & $\begin{array}{l}\text { (c) } 2021 \text { IEEE. Personal use of this material is permitted. Permission from IEEE must be obtained for all other uses, in } \\
\text { any current or future media, including reprinting/republishing this material for advertising or promotional purposes, } \\
\text { creating new collective works, for resale or redistribution to servers or lists, or reuse of any copyrighted component of } \\
\text { this work in other works. }\end{array}$ \\
\hline Tyре & article (author version) \\
\hline File Information & Litz-Wire_Final_Submission_HUSCAP.pdf \\
\hline
\end{tabular}

Instructions for use 


\title{
Analysis of Litz Wire Losses Using Homogenization-Based FEM
}

\author{
Yoshitsugu Otomo ${ }^{1,2}$, Hajime Igarashi ${ }^{1}$, Member, IEEE, Hiroyuki Sano ${ }^{3}$, and Takashi Yamada ${ }^{3}$ \\ ${ }^{1}$ Graduate School of Information Science and Technology, Hokkaido University, Sapporo 060-0814, Japan \\ ${ }^{2}$ Research Fellow of Japan Society for the Promotion of Science (JSPS), Tokyo 102-0083, Japan \\ ${ }^{3}$ JMAG Business Company, JSOL Corporation, Tokyo 104-6205, Japan
}

\begin{abstract}
This paper proposes a novel analysis method for the losses in a litz wire considering the circulating and eddy currents. In the proposed method, the macroscopic complex permeability is introduced to evaluate the eddy current loss owing to the proximity effect. Homogenization-based finite element analysis using the macroscopic complex permeability can effectively compute the proximity effect loss. The loss owing to the circulating currents is evaluated by solving the corresponding circuit equation. It is shown that the eddy current loss is dominant for the solenoidal and spiral coils without magnetic cores because of the uniform magnetic field distribution along the wire. Moreover, the magnetic reactors in which the circulating current loss cannot be ignored are analyzed using the proposed method. It is shown that the losses computed by the proposed method agree well with the measured results.
\end{abstract}

Index Terms-Circulating current, Coils, Finite element (FE) analysis, Homogenization method, Litz wire, Proximity effect.

\section{INTRODUCTION}

$\mathbf{I}_{\mathrm{w}}^{\mathrm{T}}$ T IS necessary to accurately evaluate the winding losses in litz wires for the design processes of inductors, motors, and wireless power transfer devices [1]-[4]. When these devices are operated in low- and middle-frequency ranges $(1 \mathrm{kHz}-10 \mathrm{MHz})$, the eddy current losses owing to the skin and proximity effects can significantly increase. Moreover, since strands in litz wires are electrically shorted at their terminals, the circulating current can flow between the wires owing to the magnetic induction. This current causes additional copper loss in the litz wires. When analyzing these losses by the finite element method (FEM), one has to discretize the wires into fine elements smaller than the skin depth. This results in an unacceptably large number of finite elements.

Analytical and semi-analytical approaches have been proposed to avoid discretization of the wires into fine elements [5]-[8]. These approaches introduced frequency-dependent AC loss factors to consider the skin and proximity effects. In $[7,8]$, the skin and proximity effects of electric devices with complex geometries were computed using FEM in conjunction with AC loss factors.

Homogenization-based FEM was also proposed for the analysis of litz wires [9]-[13]. In [12], the coil region was modeled as a uniform material whose eddy current loss and diamagnetic effect are expressed by the complex permeability. The homogenization method can reduce the computing cost more than conventional FE analysis because the homogenized region can be discretized without considering the skin depth. Moreover, homogenization-based FEM in conjunction with the integral equation has been proposed so that the eddy current loss owing to the twisted structure in a litz wire is considered in the macroscopic complex permeability [13]. However, in the

Manuscript received September 11, 2020; revised November 7, 2020 and March 12, 2021; accepted May 16, 2021. Corresponding author: Y. Otomo (e-mail: otomo@em.ist.hokudai.ac.jp)

Color versions of one or more of the figures in this paper are available online at http://ieeexplore.ieee.org.

Digital Object Identifier (inserted by IEEE).

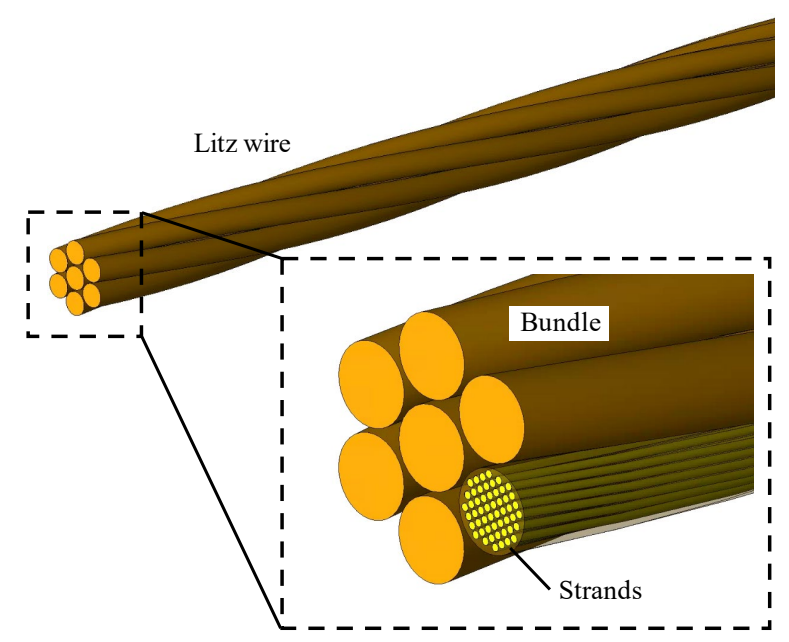

Fig. 1. Schematic of litz wire

aforementioned methods based on the AC loss factor and homogenization, the circulating current between the wires is ignored.

To analyze not only the eddy current losses but also the loss coming from the circulating current, several methods have been proposed [14]-[17]. In [14, 15], the circulating current along each strand in a litz wire was obtained by solving the partial element equivalent circuit (PEEC). However, the PEEC method requires a significant computational cost when we consider a litz wire composed of a number of strands. The zooming method that hierarchically evaluates the magnetic field distribution relaxes the problem in the analysis of litz wires [16]. However, it is still difficult to deal with litz wires composed of many strands because the zooming model has to be subdivided into fine elements of less than the skin depth. A postprocessing method based on a probabilistic approach has also been proposed in which the circulating current is computed from the magnetic vector potential obtained by the FE analysis [17]. However, this method can only deal with simply structured litz wires, which are composed of a central bundle surrounded by several bundles, as shown in Fig. 1.

In this paper, we propose a method for analyzing the litz wire 
losses using homogenization-based FEM. The macroscopic complex permeability is used to evaluate the eddy current loss owing to the proximity effect. The originality of the proposed method is that the circuit equation for the circulating current is coupled with the FE equation. The proposed method can effectively compute the eddy current losses because the litz wire composed of bundles and strands is modeled as a uniform material assuming that the circulating current in such small structures can be neglected, while the inter-wire circulating currents are considered as discussed below.

The remainder of this paper is organized as follows. In section II, we discuss the AC loss factors of the litz wire owing to the circulating current as well as the skin and proximity effects. In section III, we introduce a novel analysis method for a litz wire using homogenization-based FEM. In section IV, homogenization-based FE analysis is applied to simple solenoidal and spiral coils to discuss the influence of the circulating current. In Section V, the proposed method is applied to magnetic reactors in which the circulating current loss cannot be ignored. Finally, the paper concludes in Section VI with a concise summary of this work.

\section{AC LOSS FACTORS OF LITZ WIRE}

We consider a litz wire composed of multiple insulated strands, as shown in Fig. 1. Each bundle contains a large number of twisted strands (several hundred). Although the strands and bundles are insulated from each other, they are electrically shorted in the terminals. The litz wire shown in Fig. 1 has not only eddy current loss but also loss owing to circulating currents between strands and bundles. In this section, we discuss the $\mathrm{AC}$ loss factors in the litz wire relevant to the circulating current as well as the skin and proximity effects.

\section{A. Skin and Proximity Effects}

The skin and proximity effects are schematically shown in Fig. 2 (a) and (b). Because the current density in the strands localizes near its surface owing to the skin effect, as shown in Fig. 2 (a), the AC resistance of each strand increases with frequency. This effect can be neglected when the skin depth is sufficiently greater than the strand radius [18]. On the other hand, the proximity effect in the strands shown in Fig. 2 (b) is owing to the magnetic field $\boldsymbol{H}_{\text {ext }}$ generated by the currents in other strands. Antiparallel eddy currents $\boldsymbol{J}_{\text {eddy }}$ flow in strands so that the time variation of $\boldsymbol{H}_{\text {ext }}$ is canceled by the magnetic dipole generated by the antiparallel currents. The AC loss owing to the proximity effect becomes dominant in the entire litz wire when the strand radius is smaller than the skin depth $[12,13]$.

\section{B. Circulating Currents}

The circulating currents at each level of the litz wire are schematically shown in Fig. 2 (c)-(e). Given the magnetic flux interlinkages across the strands, bundles, and wires, the circulating currents $I_{\text {circ }}$ flow along them according to Faraday's law. Note that these are electrically connected to the terminal. If the wires are ideally well twisted and the interlinkage flux is uniform, then the circulating currents are

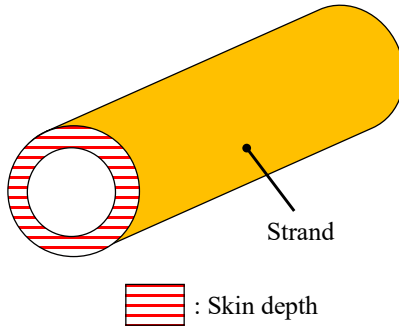

(a) Skin effect

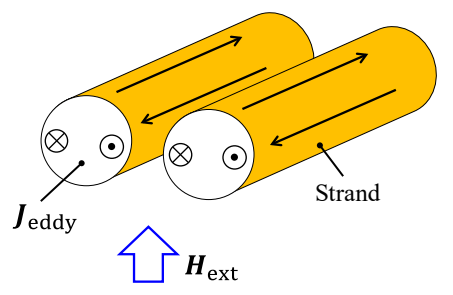

(b) Proximity effect

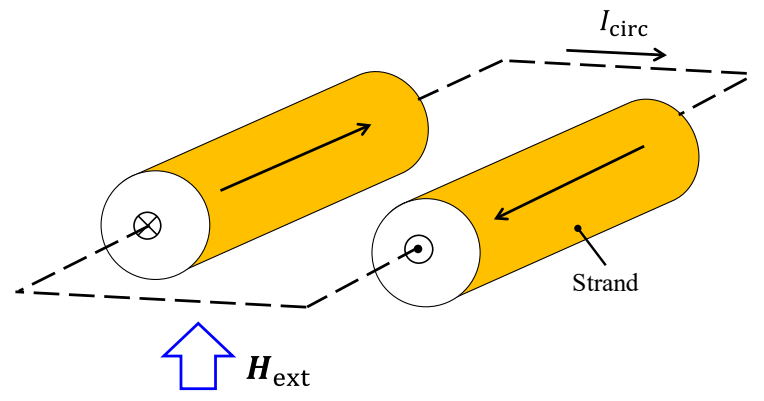

(c) Strand-level circulating current

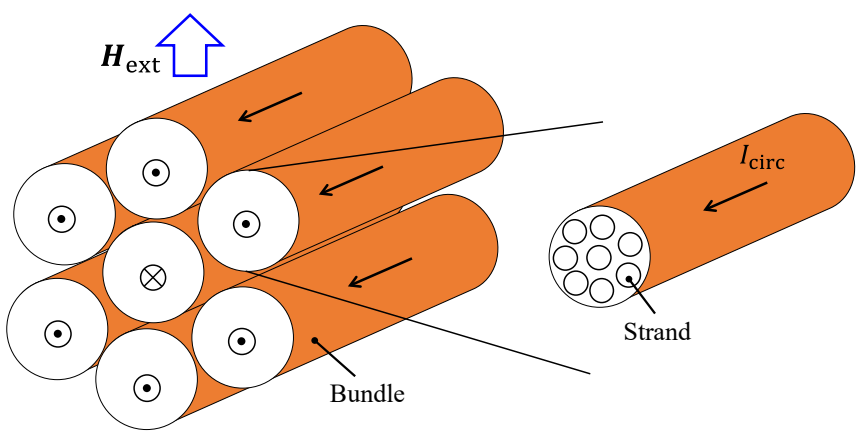

(d) Bundle-level circulating current

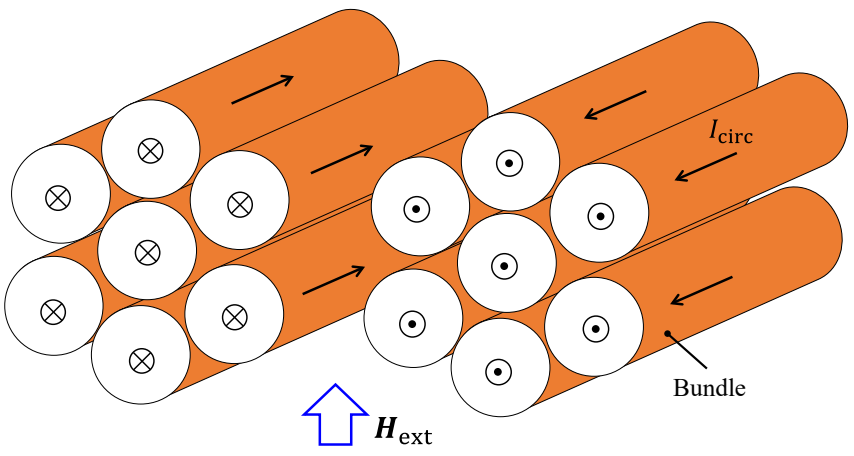

(e) Wire-level circulating current

Fig. 2. AC loss factors of litz wire

completely canceled. The twist pitch, however, is not always sufficiently smaller than the wavelength of the main Fourier component of the magnetic field. Thus, the twining does not cancel the induced voltage. For example, the central bundle shown in Fig. 2 (d) has neighboring bundles so that the current flows circularly between them [17]. These circulating currents contribute to AC losses. The relationship between the spatial profile of the magnetic field and circulating current is discussed in Appendix A. 


\section{FORMULATION}

\section{A. Complex Permeability for Proximity Effect}

The proximity effect can be effectively treated by introducing complex permeability $\dot{\mu}$. It is assumed that the magnetic field generated surrounding wires and external source is uniform in the scale of the wire radius as shown in Fig. 3. Under this assumption, the eddy current in a wire can be analytically computed. The Joule loss and diamagnetic effect coming from the eddy current can be taken into consideration by introducing the complex relative permeability $\dot{\mu}_{\mathrm{r}}[12]$

$$
\begin{gathered}
\dot{\mu}_{\mathrm{r}}=\mu_{\mathrm{r}} \frac{J_{1}(z)}{z J_{0}(z)-J_{1}(z)^{\prime}}, \\
z=a(1-\mathrm{j}) / \delta,
\end{gathered}
$$

where $\mu_{\mathrm{r}}, J_{i}(z)(i=0,1), a, \mathrm{j}$, and $\delta$ denote the relative permeability of a round wire, $i$-th order Bessel functions, wire (strand) radius, imaginary unit, and skin depth, respectively. The macroscopic complex permeability homogenized over the litz wire shown in Fig. 4 can be obtained from the extended Ollendorff formula $[12,19]$ :

$$
\langle\dot{\mu}\rangle=\mu_{0}\left\{1+\frac{2 \eta\left(\dot{\mu}_{\mathrm{r}}-1\right)}{2+(1-\eta)\left(\dot{\mu}_{\mathrm{r}}-1\right)}\right\},
$$

where $\mu_{0}$ and $\eta$ denote the vacuum permeability and volume fraction which is the ratio of the wire volume to the volume of the homogenization region, respectively. Using this permeability, we can evaluate the eddy current loss owing to the proximity effect without fine discretization of the coil region. It is remarked that the spatial distribution of the magnetic field is considered in FE analysis.

\section{B. Homogenization-Based FEM}

We analyze a coil composed of a litz wire by FEM using (2). For three-dimensional analysis, the magnetic permeability of the coil is set to $\langle\dot{\mu}\rangle$ on the cross-section plane spanned by $\tau_{1}$ and $\tau_{2}$, as shown in Fig. 4 , while it is set $\mu_{0}$ along the axial direction parallel to $\tau_{3}$. Note that the system $\left(\tau_{1}, \tau_{2}, \tau_{3}\right)$ is locally defined when the coil has curvature. Thus, we introduce the magnetic tensor $\boldsymbol{v}=\left[\begin{array}{lll}1 /\langle\dot{\mu}\rangle & 1 /\langle\dot{\mu}\rangle & 1 / \mu_{0}\end{array}\right]^{\mathrm{t}}$. When there is no conductor except the coil, we solve the magnetostatic equation given by

$$
\operatorname{rot} \boldsymbol{v}(\operatorname{rot} A)-J=0 \text {, }
$$

where $\boldsymbol{A}$ and $\boldsymbol{J}$ denote the magnetic vector potential and current density, respectively. If there are other conductors, the term $\mathrm{j} \omega \sigma \boldsymbol{A}$ relevant to the eddy current is included in (3), where $\omega$ and $\sigma$ are the angular frequency and conductivity of the conductor, respectively. Moreover, the circuit equations

$$
\frac{R_{k} z J_{0}(z)}{2 J_{1}(z)} I_{k}+\mathrm{j} \omega \Phi_{k}=V_{k}, \quad(k=1,2, \ldots),
$$

are coupled with (3) to consider the circulating currents in the

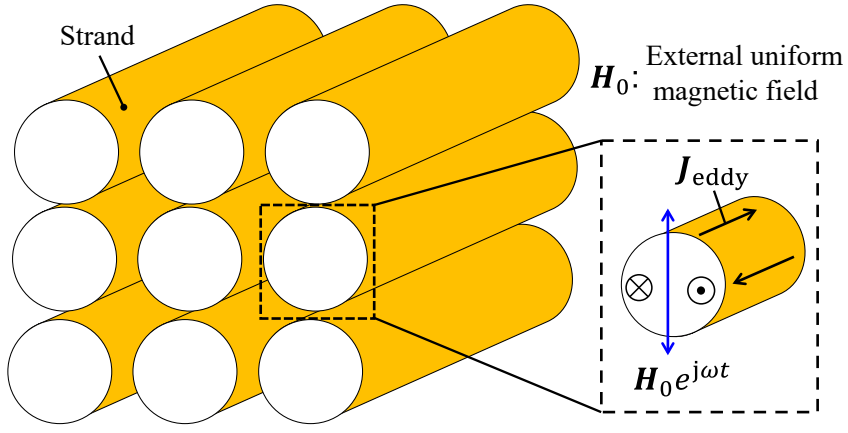

Fig. 3. Round wire immersed in time-harmonic uniform magnetic field

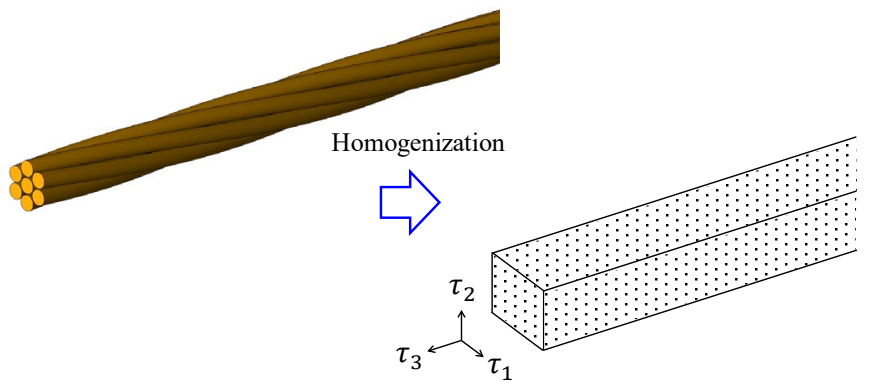

Fig. 4. Homogenization of litz wire using macroscopic complex permeability

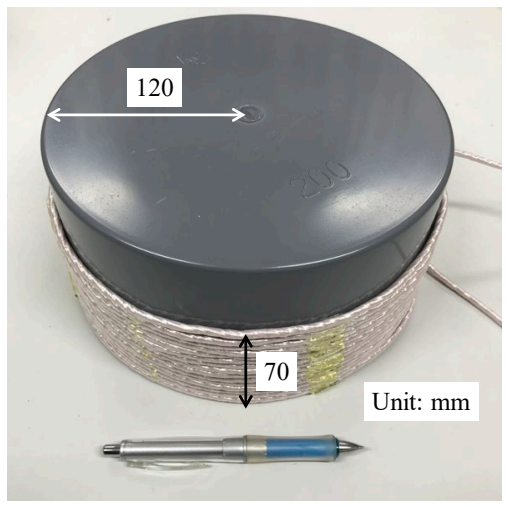

(a) Solenoidal coil

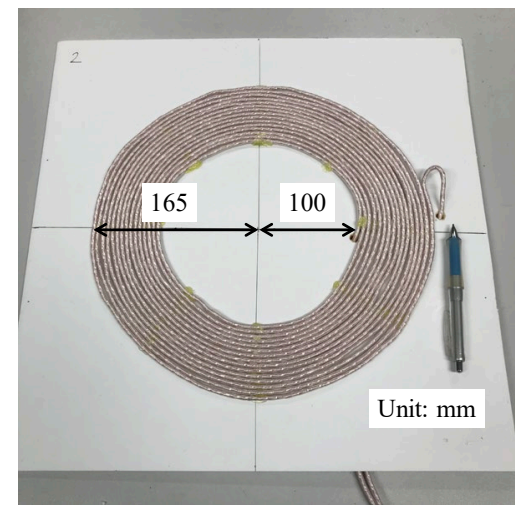

(b) Spiral coil

Fig. 5. 15 turn solenoidal and spiral coils

$k$-th litz wire, where $R_{k}, I_{k}, \Phi_{k}$, and $V_{k}$ denote the DC resistance, circuit current, interlinkage flux, and input voltage, respectively. In the first term in (4), we consider the impedance 
owing to the skin effect [12]. Note that the circulating currents are included in the circuit current $I_{k}$ together with the source current. The FE discretization of (3) and (4) leads to

$$
\begin{aligned}
& \sum_{j} A_{j} \int_{\Omega}\left(\operatorname{rot} \boldsymbol{N}_{i}\right)^{\mathrm{t}} \mathrm{T}^{\mathrm{t}} \mathbf{v} \mathrm{T}\left(\operatorname{rot} \boldsymbol{N}_{j}\right) d \Omega \\
&-\sum_{k} I_{k} \int_{\Omega_{\text {coil }}} \boldsymbol{N}_{i} \cdot \boldsymbol{j}_{k} d \Omega=0, \\
& \frac{R_{k} z J_{0}(z)}{2 J_{1}(z)} I_{k}+\mathrm{j} \omega \sum_{j} A_{j} \int_{\Omega_{\text {coil }}} \boldsymbol{N}_{j} \cdot \boldsymbol{j}_{k} d \Omega=V_{k},
\end{aligned}
$$

where $\boldsymbol{N}_{i}$ and $\boldsymbol{j}_{k}$ denote the vector interpolation function and unit current density $\boldsymbol{j}_{k}=\boldsymbol{J}_{k} / I_{k}$, respectively. Moreover, $\mathrm{T}^{\mathrm{t}} \boldsymbol{v} \mathrm{T}$ is the reluctance tensor, by which the local components shown in Fig. 4 are transformed into Cartesian components. The FE and circuit equations in (5) were solved using commercial software, JMAG-Designer.

\section{NumericAl Results: SolenOIDAL AND SpIRAL CoILS}

To investigate the influence of the circulating current loss in a litz wire, we consider the simple solenoidal and spiral coils shown in Fig. 5. Because the magnetic field is expected to be uniform along the axial direction of the coil, the effect of the circulating current is minor compared with that from the proximity effect. These coils consist of the litz wire shown in Fig. 6, whose physical specifications are summarized in Table I. Because the litz wire is composed of four bundles, each of which consists of many strands, there are inter-bundle and interstrand circulating currents. To compute the AC losses of these coils using the homogenization-based FE analysis, we discretized the coil models to the axisymmetric FE models as shown in Fig. 7.

The frequency dependences of the $\mathrm{AC}$ resistance of these coils computed by the extended Ollendorff formula are plotted in Fig. 8, where we do not consider the circulating currents. The $\mathrm{AC}$ resistance was measured with an LCR meter (HIOKI IM3523). We can see that the numerical results are in good agreement with the measured values over the frequency domain of interest, although the circulating current loss is not considered. Thus, it is concluded that the eddy current loss owing to the proximity effect is dominant when the bundles and strands are twisted with a pitch smaller than the spatial variation of the magnetic field along the litz wire. In other words, the circulating current loss can be ignored because the magnetic induction between the strands and bundles is canceled. Here, note that the proximity effect does not significantly depend on the twist pitch when $a<\delta[13]$.

\section{Numerical Results: Magnetic REACTORS}

\section{A. Magnetic Reactor with Two Parallel Litz Wires}

In the previous section, we found that the strand-level and bundle-level circulating current losses can be ignored when the magnetic field is sufficiently uniform along the litz wire.
TABLE I

SPECIFICATIONS OF LITZ WiRE FOR SOLENOIDAL AND SPIRAL COILS

\begin{tabular}{cc}
\hline \hline Number of strands & 100 \\
Number of bundles & 4 \\
Number of strands per bundle & 25 \\
Twist pitch of litz wire & $45 \mathrm{~mm}$ \\
Strand radius & $0.15 \mathrm{~mm}$ \\
\hline \hline
\end{tabular}

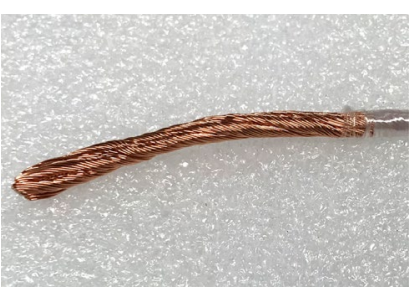

(a) Litz wire

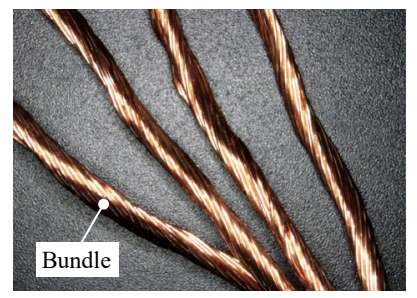

(b) Bundles of litz wire
Fig. 6. Litz wire for solenoidal and spiral coils

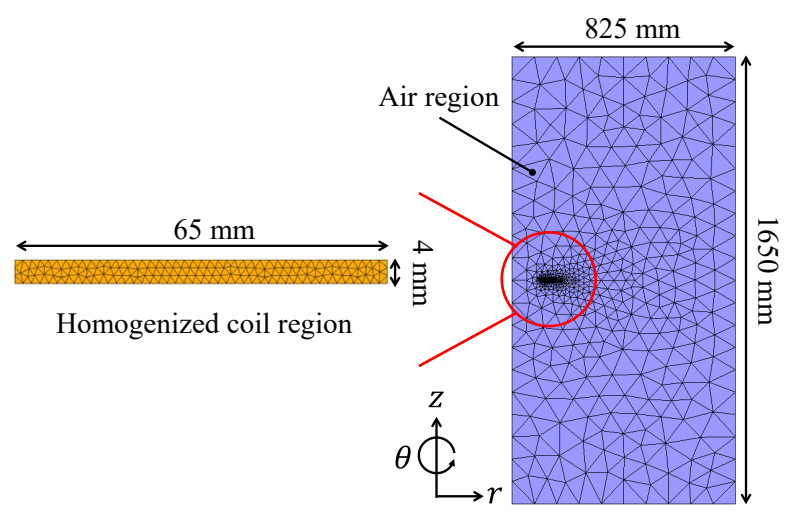

Fig. 7. Axisymmetric finite element model for spiral coil (element type: triangle, number of finite elements: 1,400 , boundary condition: Dirichlet boundary condition)



(a) Solenoidal coil

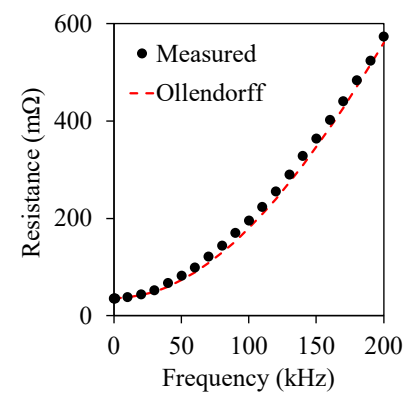

(b) Spiral coil
Fig. 8. Frequency dependence of solenoidal and spiral coils

Meanwhile, the inter-wire circulating current loss should also be considered because multilayer coils composed of litz wires are often used in electric devices to increase the quality factor. Thus, we apply the proposed method to a magnetic reactor with two parallel litz wires, as shown in Fig. 9, whose specifications are summarized in Table II.

Because there is no twist between a pair of litz wires, that is, wire 1 and 2, in the magnetic reactor as shown in Fig. 9, the inter-wire circulating current $I_{\text {circ }}$ shown in Fig. 10 can be dominant. The analysis models for the magnetic reactor with two parallel litz wires are shown in Fig. 11. 
TABLE II

SPECIFICATIONS OF MAGNETIC REACTOR WITH TWO PARALLEL LiTZ WiRES

\begin{tabular}{cc}
\hline \hline Number of strands per wire & 7 \\
Twist pitch & $20 \mathrm{~mm}$ \\
Strand radius & $0.16 \mathrm{~mm}$ \\
Length of each wire & $1000 \mathrm{~mm}$ \\
Air gap in magnetic core & $4 \mathrm{~mm}$ \\
Magnetic core type & PQ65 \\
\hline \hline
\end{tabular}
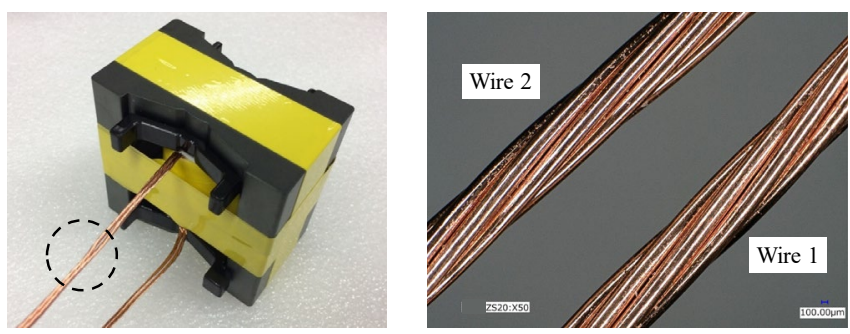

Fig. 9. 10 turn magnetic reactor with two parallel litz wires (left). Enlarged figure of litz wires in dashed circle (right)

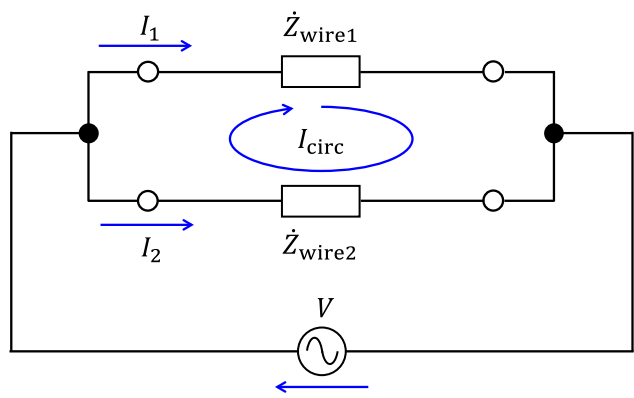

Fig. 10. Equivalent circuit of magnetic reactor with two parallel litz wires. $\dot{Z}_{\text {wire } 1}$ and $\dot{Z}_{\text {wire2 }}$ denote impedance of each litz wire.

Although the conventional homogenization model shown in Fig. 11 (a) is simpler than the proposed homogenization model shown in Fig. 11 (b), the inter-wire circulating current in each litz wire cannot be evaluated. By contrast, the proposed method allows us to evaluate the eddy current and circulating current losses because the circuit equations are solved with the field equation where the litz wires are homogenized using (2). The volume fraction $\eta$ of homogenized litz wires was set to 1.0 . In addition, the FE model for the proposed method is shown in Fig. 12.

The frequency dependences computed by the proposed method and the extended Ollendorff formula are plotted in Fig. 13 (a). It takes approximately $10 \mathrm{~s}$ for computation per sampling point using an Intel Core i7-4790 CPU (3.6 GHz, 2 cores). We can see that the resultant $\mathrm{AC}$ resistance computed by the proposed method agrees with the measured values with a maximum discrepancy of $5 \mathrm{~m} \Omega$, while the extended Ollendorff formula underestimates the measured result. To see the influence of the circulating current loss, the resistance is decomposed into contributions from the DC, proximity, and circulating currents by the proposed method, as shown in Fig. 13 (b).

We find that the resistance $R_{\text {circ }}$ owing to the inter-wire circulating current as well as the proximity effect loss $R_{\text {prox }}$

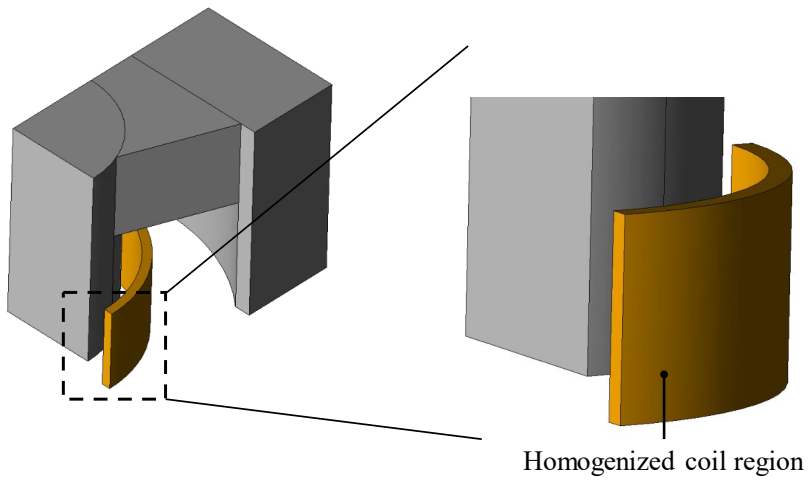

(a) Conventional homogenization model by extended Ollendorff formula

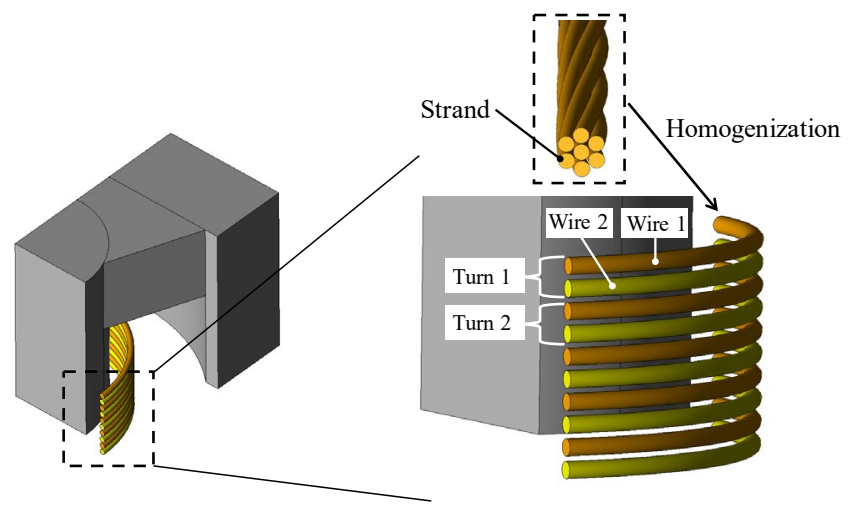

(b) Proposed homogenization model

Fig. 11. Analysis models for magnetic reactor with two parallel litz wires ( $1 / 8$ fraction is shown)

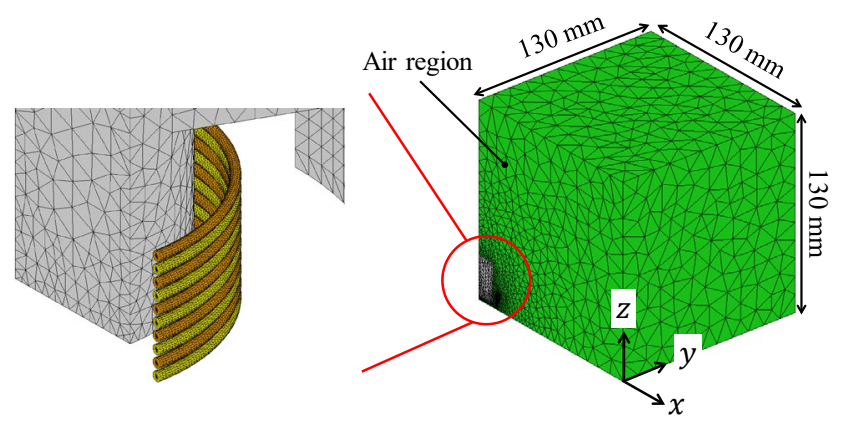

Fig. 12. Finite element model for magnetic reactor with two parallel litz wires (element type: tetrahedron, number of finite elements: 125,590 , boundary condition: $\mathrm{x}-\mathrm{y}$ plane (Neumann boundary condition), else (Dirichlet boundary condition))



(a) Simulated and measured resistance

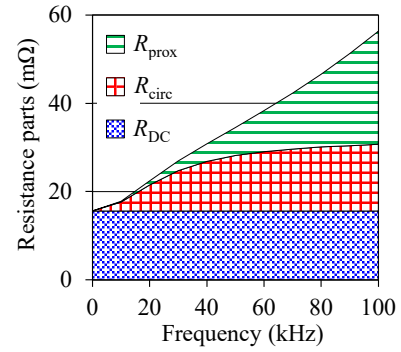

(b) Decomposed resistance obtained by proposed method
Fig. 13. Frequency dependence of magnetic reactor with two parallel litz wires 

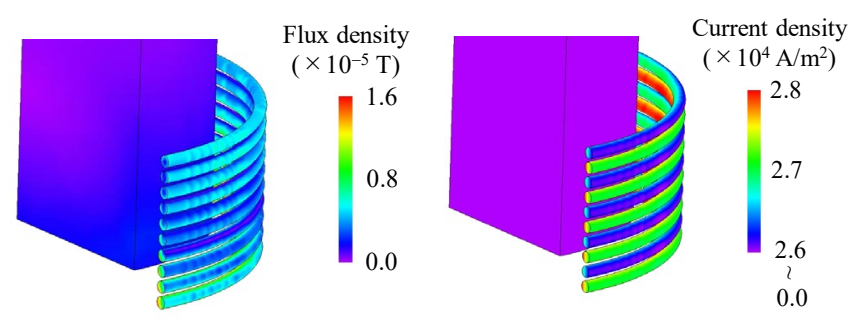

(a) Flux density (b) Current density

Fig. 14. Distribution of flux density and current density at $100 \mathrm{kHz}$ for magnetic reactor with two parallel litz wires (input voltage: $1.0 \mathrm{~V}$ )

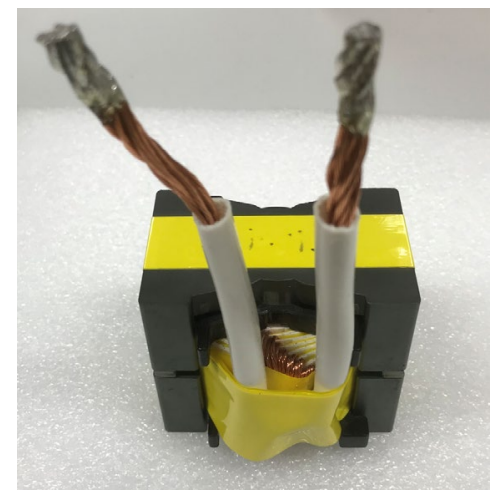

Fig. 15. 10 turn magnetic reactor with 14 parallel litz wires

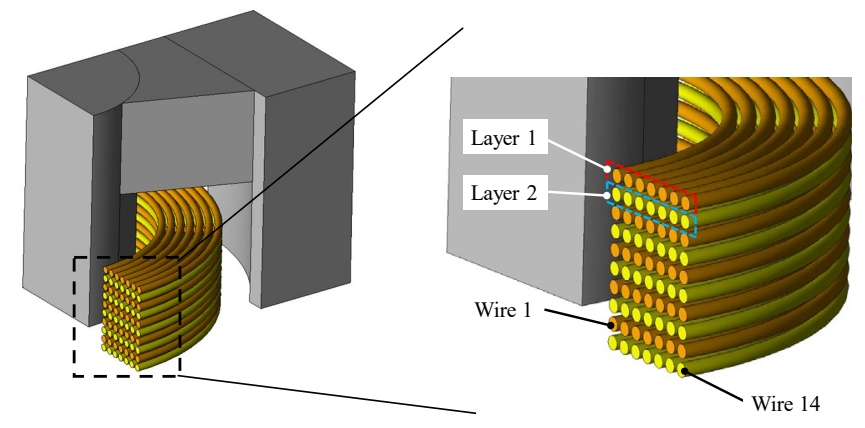

(a) Analysis model (1/8 fraction is shown)
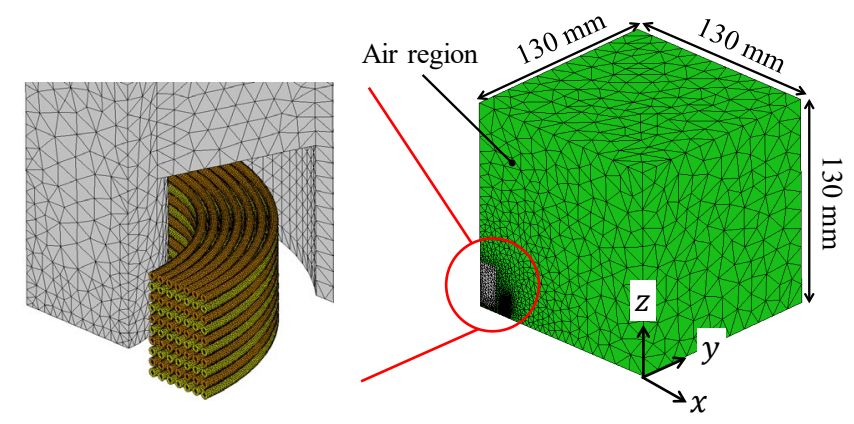

(b) Finite element model (element type: tetrahedron, number of finite elements: 804,020, boundary condition: $x-y$ plane (Neumann boundary condition), else (Dirichlet boundary condition))

Fig. 16. Analysis and finite element models for magnetic reactor with 14 parallel litz wires

make significant contributions. The reason why the circulating current loss saturates at high frequency is discussed in the Appendix B. The distributions of the flux density and current

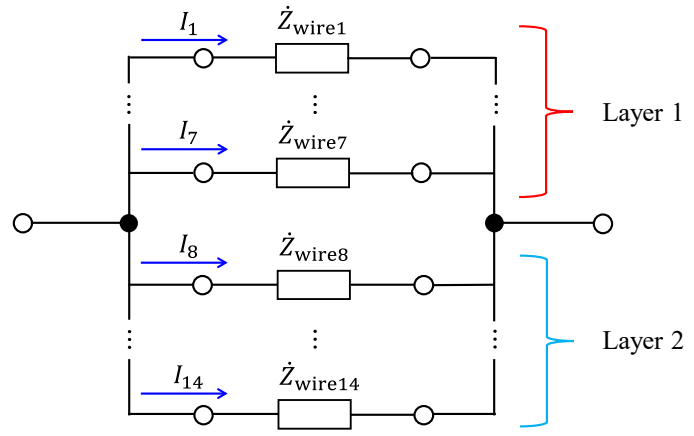

Fig. 17. Equivalent circuit of magnetic reactor with 14 parallel litz wires

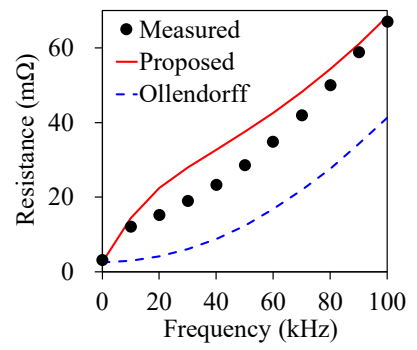

(a) Simulated and measured resistance

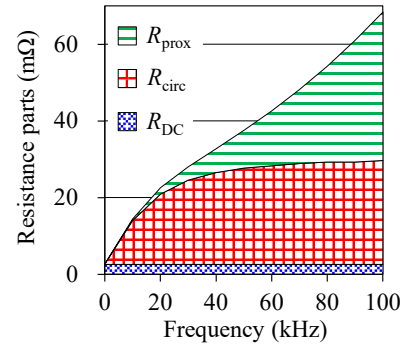

(b) Decomposed resistance obtained by proposed method
Fig. 18. Frequency dependence of magnetic reactor with 14 parallel litz wires

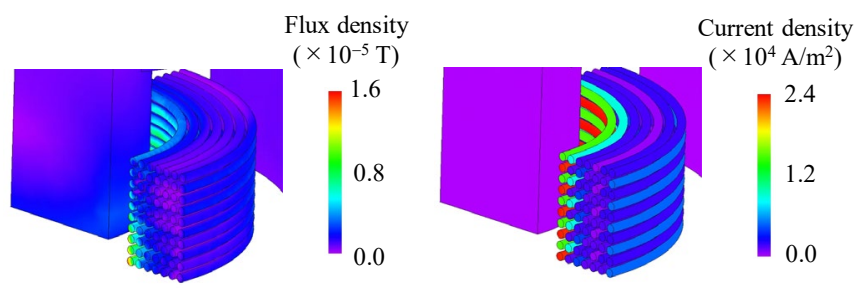

(a) Flux density

(b) Current density

Fig. 19. Distribution of flux density and current density at $100 \mathrm{kHz}$ for magnetic reactor with 14 parallel litz wires (input voltage: $1.0 \mathrm{~V}$ )

density at $100 \mathrm{kHz}$ are shown in Fig. 14. Because the circulating current between the wires flows so as to cancel the radial interlinkage flux around the air gap, the current density of each wire differs significantly. On the other hand, because the magnetic field distribution along the wire of each turn is almost uniform, the strand-level circulating current between the twisted strands can be ignored.

\section{B. Magnetic Reactor with 14 Parallel Litz Wires}

We apply the proposed method to a more practical model: a magnetic reactor with 14 parallel litz wires, as shown in Fig. 15. There are 980 stranded wires in total. Conventional FEM requires a high computing cost to evaluate the wire losses in this reactor. The analysis and FE models for the proposed method and the equivalent circuit of the magnetic reactor are shown in Figs. 16 and 17, respectively. Each layer includes seven parallel litz wires. The frequency dependence of the $\mathrm{AC}$ resistance is shown in Fig. 18. It takes approximately $90 \mathrm{~s}$ for computation per sampling point under the aforementioned computing environment. We can see that the resultant $\mathrm{AC}$ resistance 
computed by the proposed method agrees with the measured result with a maximum discrepancy of $10 \mathrm{~m} \Omega$. The magnetic field distribution in the wires is concentrated around the air gap so that the current density is significantly localized, as shown in Fig. 19. It is concluded that litz wire losses can be effectively evaluated by the proposed method.

In future studies, the validity of the proposed method should be verified when the bundles and strands are twisted with a pitch larger than the spatial variation of the magnetic field along the litz wire. In the above case, the proposed method would have to be extended so that the circulating current owing to the magnetic induction between the bundles and strands can be considered.

\section{CONCLUSION}

In this paper, we proposed a method to analyze the losses in a litz wire using homogenization-based FEM. The eddy current loss owing to the proximity effect was evaluated by the complex permeability, while the circulating current loss was directly computed from the circuit equations.

We discussed the influence of the circulating current loss using solenoidal and spiral coils. It was shown that the interbundle and inter-strand circulating current losses are sufficiently smaller than the eddy current loss owing to the proximity effect in these configurations. This is valid when the twist pitch is sufficiently smaller than the wavelength of the spatial variation in the magnetic field.

The proposed method was applied to the analysis of magnetic reactors with multilayered litz wires. It was found that the numerical results considering the eddy current and inter-wire circulating current losses agree well with the measured results. Thus, it is concluded that the proposed method is effective for the computation of losses in a litz wire.

\section{APPENDIX A}

We consider the relationship between the spatial profile of the magnetic field and circulating current. Here, the parallel and twisted wires shown in Fig. A1 are considered, where the solid and dashed lines represent two separated wires that are electrically connected at the terminals. The profiles of the magnetic field are schematically shown in Fig. A1 with the blue line.

When there exists a net magnetic flux across the parallel wires shown in Fig. A1 (a), the magnetic induction $e$ is induced along the wires according to Faraday's law. In this case, the circulating current $I_{\text {circ }}$ flows between the wires so as to cancel the interlinkage flux $\Phi$. In contrast to the parallel wires, the ideal twisted wires whose twist pitch is smaller than the wavelength of the dominant Fourier component of the magnetic field cancel out the circulating current as shown in Fig. A1 (b) since the magnetic induction of each loop is nearly equal $\left(e_{1} \approx e_{1}^{\prime} \approx e_{1}^{\prime \prime}\right)$. However, if the twist pitch is larger than the wavelength as shown in Fig. A1 (c), the magnetic inductions in the small loops do not balance. Thus, the circulating current flows in this case, and the circulating current losses of the twisted bundles and strands in the litz wire should be considered.

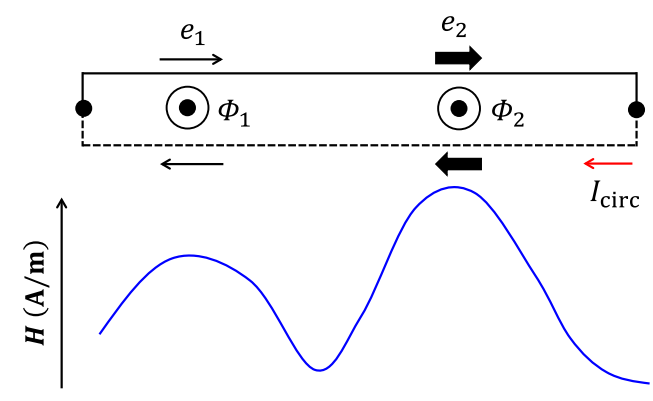

(a) Parallel wires
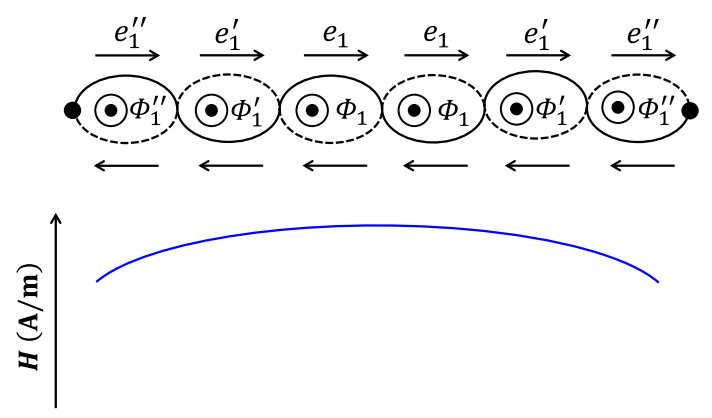

(b) Twisted wires whose twist pitch is smaller than spatial distribution of magnetic field
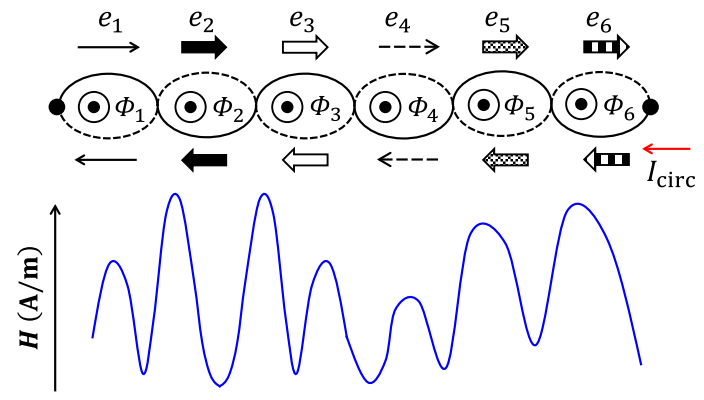

(c) Twisted wires whose twist pitch is larger than spatial distribution of magnetic field

Fig. A1. Parallel and twisted wires in each magnetic field

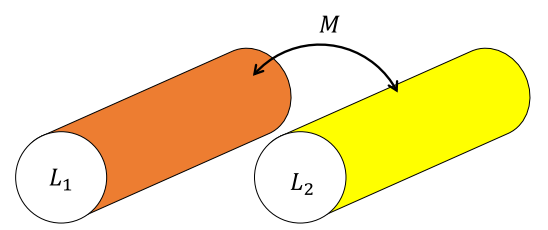

Fig. B1. Two parallel conductors

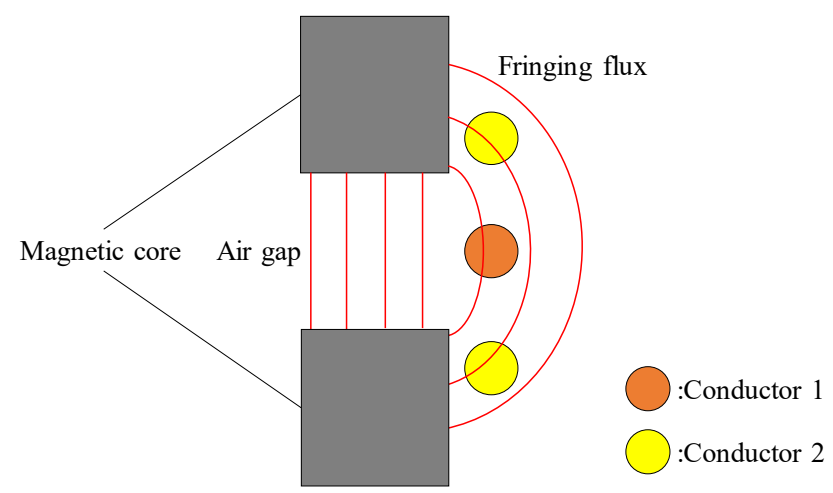

Fig. B2. Magnetic field distribution around conductors 
The systems shown in Fig. 5 would correspond to (b) in Fig. A1 where the circulating currents can be ignored.

\section{APPENDIX B}

Here, we consider the frequency dependence of the loss owing to the circulating current. For simplicity, let us consider the circulating current that flows along the two parallel conductors shown in Fig. B1. The circuit equations of the magnetically coupled conductors are given by

$$
\begin{aligned}
& \left(R_{1}+\mathrm{j} \omega L_{1}\right) I_{1}+\mathrm{j} \omega M I_{2}=V_{1}, \\
& \left(R_{2}+\mathrm{j} \omega L_{2}\right) I_{2}+\mathrm{j} \omega M I_{1}=V_{2},
\end{aligned}
$$

where $I_{i}, V_{i}, R_{i}, L_{i}(i=1,2)$, and $M$ denote the circuit current, source voltage, DC resistance, self-inductance, and mutual inductance, respectively. If these conductors are electrically shorted at the terminals, then (B1) and (B2) are rewritten as follows:

$$
\left\{R_{1}+\mathrm{j} \omega\left(L_{1}-M\right)\right\} I_{1}=\left\{R_{2}+\mathrm{j} \omega\left(L_{2}-M\right)\right\} I_{2} .
$$

From (B3), the current ratio $\kappa$ in the conductors is expressed as

$$
\kappa=\frac{I_{1}}{I_{2}}=\frac{R_{2}+\mathrm{j} \omega\left(L_{2}-M\right)}{R_{1}+\mathrm{j} \omega\left(L_{1}-M\right)}
$$

When the self-inductance values are identical $\left(L_{1}=L_{2}\right)$, the current ratio is constant regardless of the frequency. Thus, no circulating current is generated owing to magnetic induction. By contrast, the conductors have different self-inductance values $\left(L_{1} \neq L_{2}\right)$ when magnetic material affects the field distribution, as shown in Fig. B2. In this case, the circulating current flows because there can be a phase shift according to (B4) between $I_{1}$ and $I_{2}$. We consider here a simple example shown in Fig. B3 to evaluate the current ratio in (B4) due to the difference of the self-inductance between conductors. The magnetic flux distributions are shown in Fig. B4. We can see that the ununiform magnetic flux distributes due to the fringing flux around the air gap shown in Fig. B4 (b) while the magnetic flux in the coil region is almost uniform in Fig. B4 (a). The computed current ratio is plotted in Fig. B5. It can be seen that the current ratio increases as frequency when the coils are placed near the air gap of the magnetic core. Finally, to consider the frequency dependence of the circulating current at high frequencies, we take the limit of the current ratio in (B4) as

$$
\lim _{\omega \rightarrow \infty} \kappa=\lim _{\omega \rightarrow \infty}\left\{\frac{\frac{R_{2}}{\omega}+\mathrm{j}\left(L_{2}-M\right)}{\frac{R_{1}}{\omega}+\mathrm{j}\left(L_{1}-M\right)}\right\}=\frac{L_{2}-M}{L_{1}-M} .
$$

It follows from (B5) that the current ratio converges to a constant when the frequency increases, which explains the saturation in the resistance relevant to the circulating current observed in Figs. 13 and 18.

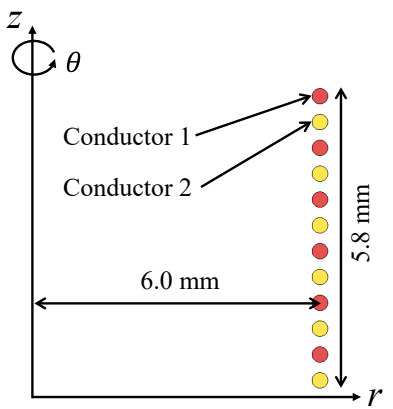

(a) Air core

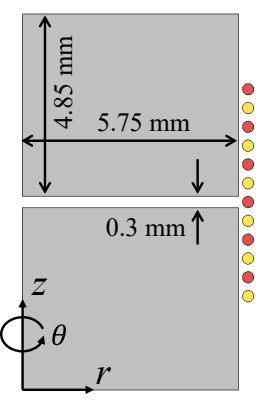

(b) Magnetic core
Fig. B3. Six turn inductors with two-parallel conductors.

(conductor radius: $0.15 \mathrm{~mm}$, relative permeability of magnetic core: 1000 )

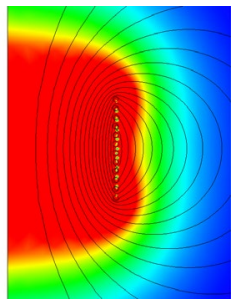

(a) Air core

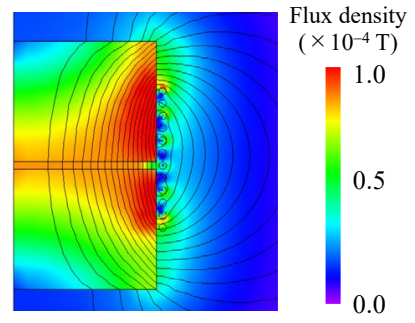

(b) Magnetic core
Fig. B4. Magnetic field distribution at $100 \mathrm{kHz}$ for six turn inductors. Note that skin and proximity effects are ignored in this example. (input voltage: $1.0 \mathrm{~V}$ )

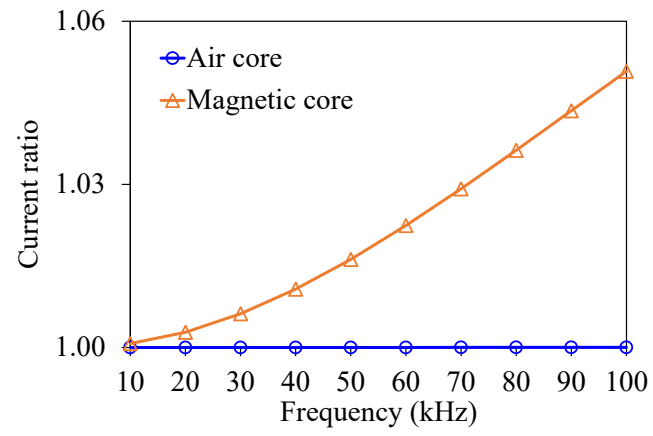

Fig. B5. Current ratio of conductor 1 to conductor 2.

\section{ACKNOWLEDGMENT}

This work was supported in part by the JSPS KAKENHI under Grants JP19J20375 and JP18H01664, and in part by the MEXT Doctoral program for the Data Related InnoVation Expert Hokkaido University (D-DRIVE-HU) Program. This work was based on the cooperative work among JSOL Co., Tabuchi Electric Co., Ltd., and Hokkaido University. The authors would like to thank Editage for editing a draft of this article.

\section{REFERENCES}

[1] R. P. Wojda, and M. K. Kazimierczuk, "Winding Resistance and Power Loss of Inductors with Litz and Solid-Round Wires," IEEE Trans. Ind. Appl., vol. 54, no. 4, pp. 3548-3557, 2018.

[2] M. Al Eit, F. Bouillault, C. Marchand, and G. Krebs, "2-D Reduced Model for Eddy Currents Calculation in Litz Wire and Its Application for Switched Reluctance Machine," IEEE Trans. Magn., vol. 52, no. 3, Art. no. $7401304,2016$. 
[3] H. Hämäläinen, J. Pyrhönen, J. Nerg, and J. Talvitie, "AC Resistance Factor of Litz-Wire Windings Used in Low-Voltage High-Power Generators," IEEE Trans. Ind. Electron., vol. 61, no. 2, pp. 693-700, 2014.

[4] C. Roth, and D. Gerling, "Novel Calculation Model for Bunched Litz Wires," in Proc. IEEE WPTC, pp. 162-165, 2019.

[5] F. Tourkhani, and P. Viarouge, "Accurate Analytical Model of Winding Losses in Round Litz Wire Windings," IEEE Trans. Magn., vol. 37, no. 1 , pp. 538-543, 2001.

[6] H. Li, N. Zhang, S. Wang, and J. Zhu, "An Analytical Loss Model of LitzWire Winding for Transformers Excited by Converters with Winding Configurations Considered," IEEE Trans. Magn., vol. 55, no. 9, Art. no. $7500405,2019$.

[7] C. R. Sullivan, and R. Y. Zhang, "Analytical Model for Effects of Twisting on Litz-Wire Losses," in Proc. COMPEL, pp. 1-10, 2014.

[8] Z. Liu, J. Zhu, and L. Zhu, "Accurate Calculation of Eddy Current Loss in Litz-Wired High-Frequency Transformer Windings," IEEE Trans. Magn., vol. 54, no. 11, Art. no. 8401605, 2018.

[9] J. Gyselinck, P. Dular, "Frequency-Domain Homogenization of Bundles of Wires in 2-D Magnetodynamic FE Calculations," IEEE Trans. Magn., vol. 41, no. 5, pp. 1416-1419, 2005.

[10] K. Niyomsatian, J. J. C. Gyselinck, and R. V. Sabariego, "Experimental Extraction of Winding Resistance in Litz-Wire Transformers: Influence of Winding Mutual Resistance," IEEE Trans. Power Electron., vol. 34, no. 7, pp. 6736-6746, 2019.

[11] Y. Sato, and H. Igarashi, "Homogenization Method Based on Model Order Reduction for FE Analysis of Multi-Turn Coils," IEEE Trans. Magn., vol. 53, no. 6, Art. no. 1100104, 2017.

[12] H. Igarashi, "Semi-Analytical Approach for Finite Element Analysis of Multi-turn Coil Considering Skin and Proximity Effects," IEEE Trans. Magn., vol. 53, no. 1, Art. no. 7400107, 2017.

[13] S. Hiruma, Y. Otomo, and H. Igarashi, "Eddy Current Analysis of Litz Wire Using Homogenization-Based FEM in Conjunction with Integral Equation,” IEEE Trans. Magn., vol. 54, no. 3, Art. no. 7001404, 2018.

[14] T. Guillod, J. Huber, F. Krismer, and J. W. Kolar, "Litz Wire Losses: Effects of Twisting Imperfections," in Proc. COMPEL, pp. 1-8, 2017.

[15] A. Roßkopf, E. Bär, C. Joffe, and C. Bonse, "Calculation of Power Losses in Litz Wire Systems by Coupling FEM and PEEC Method," IEEE Trans. Power Electron., vol. 31, no. 9, pp. 6442-6449, 2016.

[16] H. Katagiri, H. Sano, K. Semba, N. Mimura, and T. Yamada, "Fast Calculation of AC Copper Loss for High Speed Machines by Zooming Method," IEEJ Journal of Ind. Appl., vol. 6, no. 6, pp. 395-400, 2017.

[17] S. Gyimóthy, S. Kaya, D. Obara, M. Shimoda, M. Masuda, S. Bilicz, J. Pávó, and G. Varga, "Loss Computation Method for Litz Cables with Emphasis on Bundle-Level Skin Effect," IEEE Trans. Magn., vol. 55, no. 6, Art. no. 6300304, 2019.

[18] M. Leibl, G. Ortiz, and J. W. Kolar, "Design and Experimental Analysis of a Medium-Frequency Transformer for Solid-State Transformer Applications," IEEE Emerg. Sel. Topics Power Electron., vol. 5, no. 1, pp. 110-123, 2017.

[19] F. Ollendorff, "Magnetostatik der massekerne," Arch. Elektrotechnik, vol. 25 , no. 6, pp. 436-447, 1931.
Yoshitsugu Otomo received the B.E., and M.I.S. degrees in electrical engineering from Hokkaido University, Sapporo, Japan, in 2017 and 2019, respectively.

He is currently a Doctoral Student with the Graduate School of Information Science and Technology, Hokkaido University. His current research interests include computational electromagnetism and design optimization.

Mr. Otomo is currently a Research Fellow of Japan Society for the Promotion of Science.

Hajime Igarashi (Member, IEEE) received the B.E. and M.E. degrees in electrical engineering and the Ph.D. degree in engineering from Hokkaido University, Sapporo, Japan, in 1982, 1984, and 1992, respectively.

$\mathrm{He}$ has been a Professor with the Graduate School of Information Science and Technology, Hokkaido University, since 2004. His research areas are computational electromagnetism, design optimization, and energy harvesting.

Dr. Igarashi is a member of the Institute of Electronics, Information and Communication Engineers (IEICE), International COMPUMAG Society, Japan Society for Simulation Technology (JSST), Japan Society for Computational Methods in Engineering (JASCOME), and Japan Society of Applied Electromagnetics and Mechanics (JSAEM). He was the recipient of the Outstanding Technical Paper Award by The Institute of Electrical Engineers of Japan in 2016.

Hiroyuki Sano received the M.Sc. degree in graduate school of science from the University of Tokyo in 2000.

He joined JSOL Corporation in 2000 and has been working for the development of the electromagnetic simulation software JMAG. He worked as a visiting scholar in Wisconsin Electric Machines and Power electronics Consortium (WEMPEC) during 2008-2009. He works at JSOL Corporation as an application engineer.

Takashi Yamada is CTO of JMAG division at JSOL Corporation, has been head of JMAG project since he joined the company at 1987. He started his professional career as a developer of FEM code and has been involved in numerous product development projects in industries. He is now doing product design of JMAG as well as international business operation. Besides the company business, he has been working for society of IEEJ (Institution of Electrical Engineers of Japan) and serving as a member of Investigation Committee on Electromagnetic Field Analysis for Rotating Machine. 